\title{
Germanica
}

\section{Les héros ne sont plus ce qu'ils étaient}

Le thème du voyage dans quelques œuvres de la littérature norvégienne de la fin du $\mathrm{Xx}^{\mathrm{e}}$ siècle

Auch Helden sind nicht mehr, was sie früher waren. Das Reisen in einigen

Werken den norwegischen Literatur am Ende des xx.Jahrhunderts

\section{Tone Greve Gedde}

\section{OpenEdition \\ Journals}

Édition électronique

URL : http://journals.openedition.org/germanica/2202

DOI : 10.4000/germanica.2202

ISSN : 2107-0784

\section{Éditeur}

Université de Lille

\section{Édition imprimée}

Date de publication : 31 décembre 2001

Pagination : 149-159

ISBN : 9782913857063

ISSN : 0984-2632

Référence électronique

Tone Greve Gedde, «Les héros ne sont plus ce qu'ils étaient », Germanica [En ligne], 29 | 2001, mis en ligne le 20 septembre 2013, consulté le 06 octobre 2020. URL : http://journals.openedition.org/ germanica/2202 ; DOI : https://doi.org/10.4000/germanica.2202

Ce document a été généré automatiquement le 6 octobre 2020.

(c) Tous droits réservés 


\section{Les héros ne sont plus ce qu'ils étaient}

Le thème du voyage dans quelques œuvres de la littérature norvégienne de la fin du $\mathrm{xx}^{\mathrm{e}}$ siècle

Auch Helden sind nicht mehr, was sie früher waren. Das Reisen in einigen

Werken den norwegischen Literatur am Ende des xx.Jahrhunderts

\section{Tone Greve Gedde}

1 Le thème du voyage, si on examine la question un peu plus longuement, est omniprésent dans la littérature, et donc aussi dans la littérature norvégienne. Il est bien entendu présent dans les récits de voyage proprement dits, mais aussi dans les romans et autres textes littéraires. Nombreux sont les textes dont le voyage, l'idée du voyage et le rêve de voyager sont les thèmes centraux; dans la réalité, de nombreux écrivains et autres artistes scandinaves ont quitté leur pays, surtout pour la France et l'Italie, souvent pour mieux voir ce qu'ils avaient quitté. Les pièces de Henrik Ibsen auraient-elles vu le jour sans son séjour à l'étranger? Ou pris la forme qu'elles ont prise? Osvald des Revenants compare la liberté qu'il a trouvée à Paris à l'atmosphère étouffante de son village natal. Des générations de personnages de roman et de théâtre ont exprimé des sentiments similaires. Pourtant, à la fin du XIX ${ }^{\mathrm{e}}$ siècle, à l'époque où les pièces de Henrik Ibsen sont jouées sur toutes les scènes d'Europe, au moment où Knut Hamsun publie son roman Sult (La Faim, 1890), qui sera par la suite considéré comme une œuvre majeure de la littérature moderne, quels sont les plus grands succès de librairie en Norvège? Ce sont les récits de Fridtjof Nansen, dans lesquels il conte sa traversée du Groenland à ski (1880), puis sa tentative d'atteindre le pôle Nord. Quel est probablement le plus grand best-seller norvégien de l'après-guerre ? C'est une œuvre de Thor Heyerdahl, Le voyage du Kon-Tiki (1948), qui raconte la traversée du Pacifique par une poignée d'hommes sur un radeau. Le film qui en a été tiré a par la suite connu un très grand succès commercial, couronné par un « Oscar » en 1952.

2 La popularité de ces récits est sans doute à rapprocher du rôle actif qu'ils ont joué dans la définition de l'image que les Norvégiens se font d'eux-mêmes, une image 
délibérément construite tout au long $\mathrm{du} \mathrm{xx}^{\mathrm{e}}$ siècle, l'époque où se cristallise en fait l'idée moderne de la nation norvégienne. Cette idée se fonde entre autre sur l'image du Viking, homme dur et intrépide qui maîtrise aussi bien les éléments que ses adversaires, voisins et étrangers. Les expéditions des Vikings vers les pays européens, l'Islande, le Groenland et l'Amérique, et le succès des explorateurs norvégiens en compétition farouche avec les explorateurs d'autres pays à la fin du XIXe et au début du $\mathrm{xx}^{\mathrm{e}}$ siècle, font partie intégrale de la mythologie nationale norvégienne. Ils trouvent un public enthousiaste dans un pays où la survie a souvent dépendu de la résistance physique, où l'effort est un objet d'admiration. Thor Heyerdahl, un autre aventurier dans l'âme, prouve aux Norvégiens qu'ils n'ont rien perdu de cet esprit d'aventure.

\section{Voyager, c'est quitter quelque chose et chercher quelque chose}

3 Le voyage est, bien entendu, le cadre généralement utilisé dans les romans d'éducation. Le voyage réel, physique, extérieur, est le pendant du parcours et du développement intérieurs du jeune héros. Il n'est dès lors pas étonnant que ce thème se retrouve dans la littérature pour enfants et adolescents et dans les contes folkloriques, deux autres genres où le thème du voyage est central et souvent à la fois l'image et le moteur du parcours suivi par le caractère principal. Le thème « au foyer - sans foyer - retour au foyer » est celui de la rupture avec un monde idyllique et maîtrisé. Le personnage se voit projeté hors de chez lui, dans un monde plein d'obstacles qu'il faut surmonter, que le héros parvient finalement à ramener au calme, à l'ordre et à l'harmonie, mais cette fois à un degré supérieur de sagesse et de maturité. Les récits de Nansen et de Heyerdahl, dont les voyages avaient d'ailleurs des fins scientifiques, sans être des textes littéraires proprement dits, sont eux aussi l'expression d'une évolution et d'une maturation, lorsqu'ils content les exploits et la lutte pour la survie du héros qui affronte les forces de la nature. L'expérience acquise pendant le voyage contribue au développement des participants, non seulement sur le plan physique, mais aussi par sa dimension mentale, par la preuve qu'elle leur donne de leur endurance. Si c'est peutêtre le côté aventureux qui explique le mieux la fascination qu'ont suscitée ces voyages, il n'en reste pas moins qu'un voyage mené à bien signifie aussi que le projet du personnage principal a été couronné de succès. L'objectif de l'équipe scientifique est atteint. Nous constatons une fois de plus une similitude avec le roman d'éducation.

Des aventuriers, hommes et femmes, partent encore aujourd'hui en expédition dans les régions polaires. À leur retour, ils publient naturellement le récit de leur voyage. Trouvent-ils encore la même audience auprès du grand public? Ils sont bien sûr fêtés et admirés, mais ne trouvent peut-être plus le même écho que par le passé. Ces expéditions sont avant tout perçues comme des prouesses personnelles, même si certaines expéditions ont eu pour propos avoué de servir d'inspiration et d'exemple à des tiers. Ces voyages de l'extrême ne semblent pas avoir marqué la création littéraire. Il existe en revanche d'autres manières de traiter du voyage, qui paraissent plus caractéristiques de la littérature contemporaine. D'innombrables romans ont décrit depuis plus de cent ans le déracinement des fils de paysans partis faire des études en ville. Un voyage effectué par de nombreux Norvégiens dans la seconde moitié $d u x^{e}$ siècle, à savoir la migration vers les villes et les grands centres urbains, a plus récemment été brillamment illustré par Roy Jacobsen dans Seierherrene (Les 
vainqueurs), roman paru en 1991. Trois romans publiés en 1974, 1987 et 1999 et qui ont trouvé de nombreux lecteurs, reflètent à mon avis de façon assez significative l'époque dans laquelle ils ont été écrits et pourront ici servir d'exemple.

\title{
Kjærleikens ferjereiser (1974) par Edvard Hoem
}

\author{
(Les ferries de l'amour)
}

\section{Voyages sans aventure}

Ce roman collectif, post-1968, est loin de mener le lecteur vers des horizons lointains. Conformément au canon du réalisme social, le voyage se réduit ici aux courtes traversées effectuées entre le continent et les communautés de l'archipel qui borde la côte. Ces petits voyages sur les ferries de la côte Ouest du pays rythment pourtant une éducation et un réveil des voyageurs, la prise de conscience naissante d'une population de gens ordinaires de la fin des années soixante qui s'interrogent sur la place qui leur est faite et sur leur rôle dans leur communauté locale et dans la société contemporaine. À cet égard, l'utilisation du thème du voyage est dans une certaine mesure comparable à celle que l'on trouve dans les romans d'éducation. Comme le titre l'annonce, le va-etvient quotidien et somme toute trivial entre la ville et les îles de l'archipel sert à symboliser les rapports entre ce qui est social et ce qui est personnel, entre les sphères publique et privée. L'œuvre décrit le rôle des bacs sans lesquels la survie de ces petites communautés serait impossible, la séparation et les retrouvailles physiques et sentimentales des personnages. Un aspect important du roman est le rôle de l'État providence dans le modelage de la société tel qu'il est perçu par plusieurs personnages du roman, qui y voient la trahison de la classe ouvrière par le parti travailliste norvégien, scellée entre autre par la décision de ne plus assurer la liaison par ferry.

La Norvège a connu comme le reste de l'Europe une radicalisation postsoixantehuitarde de sa jeunesse, et une bonne partie des jeunes écrivains, dont Edvard Hoem, ont souhaité contribuer par leurs œuvres à une prise de conscience politique chez leurs lecteurs. Kjorleikens ferjereiser est un des romans de cette période de réalisme social qui mérite encore d'être lu pour ses qualités littéraires, et en tant que reflet de son époque. Ironiquement, ou peut-être précisément pour cette raison, cette œuvre n'est pas typique pour cette forme d'expression littéraire : il expérimente avec la forme romanesque, rompt avec les canons réalistes. Le narrateur, "celui qui écrit ", se trouve à Oslo. Pour parler véridiquement de l'Ouest du pays, il réarrange la côte selon ses besoins, il dessine sur la carte une communauté locale fictive. Très vite, il s'aperçoit qu'il ne peut pas dans le même temps habiter la capitale et dire la vérité sur le littoral occidental du pays, il doit s'y rendre en personne, voir de ses propres yeux. Il traverse donc la Norvège d'est en ouest pour rencontrer ses propres personnages de roman, et peu à peu se faire duper par eux. L'auteur remet ainsi en question le rôle de l'auteur et le rapport entre fiction et réalité. La fiction sera aussi vraie que la réalité. 


\title{
Bortførelsen (1987) par Mette Newth \\ (Les Voleurs d'hommes, Gallimard 1994)
}

\author{
Roman pour adolescents
}

\section{Le revers des expéditions européennes}

7 Un navire hollandais, son capitaine anglais et son équipage en partie norvégien affrétés par des marchands de Bergen, partent pour explorer le Groenland et trouver le passage du nord-ouest vers l'Inde. Le roman se compose des récits alternés de deux jeunes filles, Osuqo l'Inuit et Christine la Norvégienne.

Osuqo et son fiancé Poq sont enlevés par les Européens et ramenés prisonniers à Bergen, pour y être traités comme des animaux rares et dangereux. Ils sont considérés comme des créatures diaboliques, une menace qu'on a le droit - au nom du christianisme - de traiter sans pitié.

Christine, servante chez un des riches marchands, est nommée geôlière de la jeune captive. Christine est la fille du seul marin du navire qui a tenté de défendre Osuqo lors de son enlèvement. Il a lui-même péri dans le tumulte. Christine voit ce navire noir et menaçant revenir du voyage qui lui a ravi son père adoré, son seul soutien au monde, et ramener deux étrangers qu'on dit enfants du diable et qui sont pour elle la cause de la perte qu'elle a subie. Elle est remplie de crainte, d'horreur et de haine, sentiments qui céderont avec le temps la place à la pitié, à la compréhension et à la sympathie.

10 Osuqo, après son enlèvement et son viol collectif par les marins, sombre dans la dépression et la maladie, elle voyage dans le temps, revit son passé dans son délire. C'est ainsi que nous est contée la vie des Inuits, un peuple vivant en harmonie avec la nature. L'image de la société groenlandaise est fortement structurée par la description de la nature et des relations entre hommes et nature. Soleil, lumière, glace, vent contrastes violents, changements perpétuels - laissent une impression de liberté, de beauté, de bonheur. Remarquons au passage que la nature joue très souvent un rôle positif dans la littérature nordique. Cette société s'oppose point pour point à celle de Bergen, qui nous apparaît à travers la vie de Christine, pauvre servante travaillant dur pour un maître sans cœur. L'atmosphère de Bergen est donnée pour sombre, oppressante et caractérisée par une absence totale de nature : la ville pue, elle écrase les hommes. Quant à la société, loin de proposer un cadre libérateur, elle enferme les hommes dans le carcan des hiérarchies sociales.

$11 \mathrm{Au}$ Groenland, les hommes vivent en harmonie avec la nature, leur vie et leur survie suivent et dépendent des rythmes saisonniers. Ils tirent leur subsistance de la pêche et de la chasse, mais sans porter atteinte à l'équilibre naturel. Les hiérarchies sociales attribuent à chacun sa place, mais sont aussi garantes de la liberté et de l'égalité. Comme dans toute société qui dépend étroitement de la nature, se pose aussi la question des faibles. On apprend aux enfants tout ce dont ils ont besoin pour pouvoir maîtriser plus tard leur environnement, on leur accorde une attention sans faille et on leur donne la confiance en eux dont ils ont besoin pour survivre et vivre librement. Mais venu le temps où certains deviennent trop vieux, trop faibles pour contribuer à la survie du groupe, ces personnes choisissent elles-mêmes l'heure de leur mort. Ainsi est admirablement contée la mort des grands-parents d'Osuqo, qui mettent fin à leurs jours par l'œettespranget, c'est-à-dire en se jetant du haut d'une falaise. Cette façon de 
mettre fin à ses jours, ce voyage sans retour, a souvent été décrit avec horreur et mépris par les missionnaires, qui y voyaient la preuve de la cruauté des sociétés primitives. L'auteur choisit de le présenter sous un jour positif, comme le choix nécessaire des Anciens, réalisé de plein gré pour que la tribu puisse survivre.

À travers le récit des jeunes filles et la description de la place qu'elles occupent dans leurs communautés respectives, est tracé à l'aide de parallèles et de contrastes le portrait de deux formes de sociétés différentes.

13 Et quand à la fin Osuqo libère Poq pour fuir avec lui de Bergen vers la mer et vers une mort plus que probable, leur dernier voyage est perçu plus comme une libération que comme une fin tragique. La mer ensoleillée s'ouvre à eux, les accueille en son sein. Christine, qui est au fond la véritable prisonnière, s'enferme dans un placard pour ne plus rien voir, ni entendre. Christine est « la seule victime» (p. 200) car elle n'a jamais connu et ne connaitra d'ailleurs jamais la liberté. Mais Osuqo lui a permis d'entrevoir son existence.

14 Le roman a été inspiré par un tableau du xvII siècle représentant une jeune fille groenlandaise qui fut réellement enlevée du Groenland et amenée à Bergen. Le roman fait aussi appel à d'autres documents historiques authentiques. Cette façon de présenter les deux sociétés n'est pas équilibrée. L'auteur choisit consciemment une présentation favorable à la société traditionnelle groenlandaise, celle d'un peuple vivant en harmonie avec la nature - politiquement correcte, écologiquement correcte, peut-être naïve ou simpliste pour ce qui est de l'image du bon sauvage. Cette approche s'inscrit dans le cadre du roman pour la jeunesse, généralement plus tranché noir sur blanc que les romans pour des lecteurs adultes. Écrire pour la jeunesse est un choix délibéré de l'auteur, qui la juge plus ouverte, plus réceptive et estime que ses valeurs détermineront notre avenir. La trame du récit n'est pas étrangère à nos contacts culturels actuels, à l'émigration vers l'Europe de personnes venues d'autres continents et à la rencontre d'autres expressions culturelles, croyances et modes de vie. Le roman est à l'évidence conçu comme une contribution à un réexamen des valeurs des explorateurs européens et de leurs voyages, mais veut aussi dans le même temps promouvoir la compréhension et le respect interculturels dans la société d'aujourd'hui.

Malgré un message qu'elle ne fait rien pour masquer et que confirme par ailleurs une postface de l'auteur, l'œuvre est une expression artistique de haute tenue littéraire. Elle est populaire auprès des jeunes, souvent utilisée à l'école et traduite dans une vingtaine de langues. Elle suppose pourtant un retournement complet de la perspective. L'image que nous avons du voyage se retourne contre nous, il n'est plus l'expression d'une quête libératrice d'horizons plus ouverts; le regard de l'autre nous propose une autre version. Ceci bien sûr n'est pas nouveau; d'autres auteurs ont consacré des œuvres critiques aux voyages des Européens, comme par exemple le récit par Thorkild Hansen des expéditions dano-norvégiennes aux $\mathrm{XVII}^{\mathrm{e}}$ et $\mathrm{XVIII}^{\mathrm{e}}$ siècles. Nansen et Heyerdahl ne sont pas représentatifs de ces expéditions de "négriers", mais tous ces récits d'expéditions sont parés du lustre de l'aventure. Le roman de Newth choque: des Groenlandais retenus prisonniers en Norvège... Ce n'est pas sous ce jour que nous avons jusqu'ici vu nos explorateurs. Les canailles étaient toujours les autres. Mette Newth écrit avec détermination et lance un message qu'elle juge important de faire passer. Son récit est fondamentalement différent du troisième livre que je souhaite commenter. 


\section{$L$ (1999) par Erlend Loe}

\section{Autres temps, autres mœurs?}

L'auteur, né en 1969, est le représentant d'une nouvelle génération, à en juger par son succès auprès des jeunes, succès dû surtout au roman Naiv. Super (Naïf. Super) paru en 1996. Il s'impose aussi comme chroniqueur, écrivain de scénarios pour le cinéma et auteur de livres pour enfants. Il séduit les enfants de ceux qui furent la jeunesse de 1968, de ceux qui se sont révoltés contre les valeurs établies et ont accompagné un changement considérable de la société norvégienne, jadis un pays de gens travaillant dur pour survivre et comptant désormais parmi les pays les plus riches du monde. Pour leurs enfants, toutes les portes sont théoriquement ouvertes, sauf qu'ils ne savent pas laquelle choisir, et d'ailleurs à quoi bon ? Tous les exploits ont déjà été réalisés, quelles sont les découvertes qu'il leur reste à faire?

Et il (Marco Polo) vivait dans un autre monde que le mien. Un monde inexploré, mystérieux et ouvert (p. 9).

Nous qui n'avons pas construit la Norvège. C'est nous (p. 24).

Les gens ont déjà pensé ces pensées (p. 122).

$L$ est l'histoire d'une bande de copains qui, emmenés par Erlend, l'auteur, partent en expédition sur Manuae, une des îles Cook dans le Pacifique, pour tester la véracité d'une théorie imaginée par Erlend alors qu'il faisait du patin à glace sur un lac gelé près de Trondheim, sa ville natale. Comment les habitants des îles du Pacifique sont-ils arrivés jusqu'à elles? Ne serait-il pas possible que cette partie du globe ait été à un moment donné couverte par la glace, comme l'était l'hémisphère nord ? Peut-être les habitants sont-ils arrivés là en se déplaçant sur la glace dans des temps très anciens. Dans ce cas, ne devrait-il pas être possible de retrouver leurs patins? Cependant si le roman multiplie les références aux expéditions de Nansen et de Heyerdahl, le projet d'Erlend paraît au départ nettement plus improbable. Le roman se présente comme un mélange de fiction et de documentaire. Les participants sont réellement partis pour les îles Cook. Les membres de l'expédition sont les vrais amis d'Erlend. Le narrateur du roman, Erlend, ressemble à s'y méprendre à l'auteur Erlend Loe, mais le livre est explicitement présenté comme un roman. Au lecteur de démêler où s'arrête la réalité et où commence le roman. Ce qui n'est peut-être pas si important.

Erlend Loe avait passé un contrat avec le quotidien Dagbladet et lui envoyait régulièrement des rapports publiés par le journal. L'œuvre est complétée par des photos en couleurs prises lors du voyage, et les contacts pris avec entre autre la cour de Norvège et l'Explorers Club de New York sont documentés. Le récit du séjour proprement dit n'a rien de sensationnel. Chaleur, coups de soleil, ennui et conflits pour des bagatelles. Les participants sont las de manger du poisson, meurent d'envie d'une cigarette, de café, de bière une fois que leurs stocks se sont épuisés. L'expédition prend fin principalement parce que les participants en ont assez et constatent, comme ils le savaient déjà, qu'il n'y a plus rien à découvrir. Leurs recherches se limitent à creuser quelques vagues trous dans le sable, et restent comme on peut s'y attendre sans résultat. Les voyageurs ne sont pas partis poussés par une irrésistible nécessité intérieure, mais parce qu'ils peuvent aussi bien partir pour le Pacifique que faire autre chose. Sur la fin du séjour, quand ils constatent que l'expédition n'est en fin de compte pas parvenue à ses fins, un des participants déclare : «Il est très décevant de devoir changer de rêve, mais ça passe très vite. J'en ai déjà fait l'expérience » (p. 442). Il avait 
de plus cru «- que nous pourrions faire nos recherches en un seul bon coup de collier. C'est ce que je fais depuis des années à l'université, et ça marche à chaque fois » (p. 443). Certains estiment que le «bon coup de collier » est une des caractéristiques du caractère national norvégien, ou en tout cas d'une partie de la jeunesse actuelle.

Le livre est pourtant pourvu d'un index dans la meilleure veine scientifique où les entrées

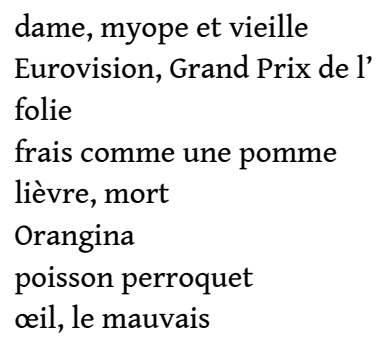

se mêlent à

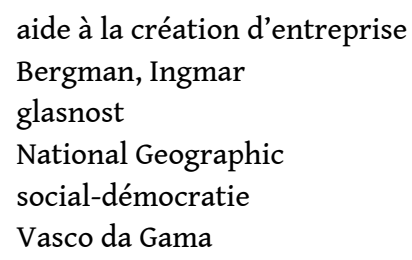

21 La plus révélatrice de leurs expériences est peut-être le test pratique des différentes formes d'organisation sociale: oligarchie, despotisme éclairé, conservatisme, communisme, capitalisme et libéralisme de marché, socialisme, social-démocratie, anarchisme, qui ont toutes leurs défauts. La social-démocratie semble l'emporter: «Elle est un peu ennuyeuse, mais elle marche » (p. 394). Une conclusion finalement fort éloignée de celle tirées par leurs parents vers 1970 et que Hoem développe dans Kjorleikens ferjereiser.

Cette expérimentation nous montre peut-être que le vrai défi est de vivre ensemble. C'était aussi un des objectifs de l'expédition du Ra en 1969 où Thor Heyerdahl voulait prouver que des personnes venues d'origines différentes pouvaient coexister dans des conditions difficiles et collaborer. Et pourtant, ces jeunes hommes ne font pas d'efforts sérieux pour tenter de communiquer avec les deux auxiliaires locaux qui les accompagnent. Leur perspective se limite au groupe de copains, une dénonciation sans doute du nombrilisme de ces jeunes. À la lumière de l'intention déclarée de Heyerdahl et de Newth de promouvoir la compréhension et la communication, ces jeunes paraissent pourtant sous un jour peu reluisant. Leur attitude peut aussi faire penser au roman Vendredi ou les limbes du Pacifique de Michel Tournier.

La plupart des critiques ont le sentiment que la légèreté apparente du roman cache une réelle gravité, que le propos de l'auteur ne se limite pas à ironiser sur lui-même et sur ses contemporains, mais ils restent hésitants. Le titre $L$ doit signifier learning, ou lering en norvégien, c'est-à-dire apprentissage. De quel apprentissage s'agit-il ? Au début du livre, Erlend philosophe: "Je considère la vie des hommes sur Terre comme un exercice interminable de travail en groupe. Le sujet, formulé dès l'aube des temps, est toujours le même: Expliquez ce qui vous entoure. Déterminez quelle est la meilleure façon de vous organiser et de tirer parti de la nature. Décrivez avec vos propres mots comment tout se tient et pourquoi vous êtes ici. Prenez le temps qu'il vous faut.» (p. 29). 
24 Le but du voyage est quand même de découvrir quelque chose sur soi-même. Le narrateur de l'ouvrage n'est absolument pas certain d'avoir découvert quoi que ce soit. À l'inverse de Knut Hamsun qui déclare dans un de ses poèmes : «- dans cent ans, tout sera oublié » pour replacer nos propres efforts dans une perspective plus large, Erlend fait confiance aux chercheurs du futur pour trouver un sens. L'œuvre se termine par la phrase : «Dans cent ans, on saura tout là dessus. On saura alors qui nous étions. » Cette dernière phrase montre que la quête identitaire est une question essentielle, aussi pour les jeunes auteurs contemporains. Dans un monde « où tout a été découvert », il n'est pas devenu plus facile de partir à la recherche de sa propre identité, au contraire peutêtre.

La métaphore du voyage n'a ainsi pas disparu de la littérature. L'absence d'une conclusion univoque n'apporte pourtant que des réponses ambiguës. Erlend et ses camarades sont revenus au foyer, mais - à la différence des héros de contes de fées sans avoir découvert grand-chose ni conquis «la princesse et la moitié du royaume». Le livre se termine par quelques mots consacrés à l'ironie (p. 454). L'approche ironique est aussi omniprésente dans cette œuvre. La question est de savoir pourquoi. N'est-il plus possible aujourd'hui de poser les questions éternelles sans s'en distancier par l'ironie? On peut y voir un signal révélant que ce récit de voyage ludique, humoristique, léger et parfois longuet n'est pas ce qu'il prétend être au premier abord. On peut en fait le soupçonner de cacher un authentique sérieux.

\section{Notre voyage commun}

Dans tous ces romans, l'aventure et les efforts hérö̈ques du voyageur ne sont plus au cœur du récit. Ces œuvres sont chacune à leur manière symptomatiques de l'époque qui les a vues naître. Le réalisme social s'intéresse au vécu et aux conditions d'existence de gens ordinaires pour mieux mettre en lumière les vrais vecteurs du pouvoir dans la société. La prise de conscience par une partie importante de la jeunesse des années 1970 et 80 des exactions commises par les Européens envers d'autres peuples, la menace d'un retour au racisme et à la xénophobie à notre époque poussent des auteurs à s'engager pour plus de respect et de compréhension entre les peuples. L'ambivalence d'un grand nombre de jeunes contemporains, à la fois hésitants et sûrs d'eux-mêmes, qui constatent que le voyage d'aventure était possible pour les héros du passé mais fait désormais partie du fonds mythologique, peut laisser une impression plus pessimiste. Il y a bien eu prise de conscience, mais le monde est resté le même. Il est pourtant possible de conclure que le thème du voyage tel qu'il est utilisé dans ces œuvres s'inscrit toujours dans la même perspective éducative et formatrice des romans d'éducation et semble toujours proposer un cadre fructueux à la prise de conscience et à la quête identitaire. 


\section{BIBLIOGRAPHIE}

Hoem, Edvard : Kjœrleikens ferjereiser, 1974.

Newth, Mette : Bortførelsen, 1987 (Les Voleurs d'hommes, Gallimard 1994).

Loe, Erlend : L (1999), Naiv. Super (1996).

Hamsun, Knut : Sult, 1890 (La Faim), Det vilde Kor (poèmes 1904).

Hansen, Thorkild : Slavernes kyst (1967), Slavernes skibe (1968), Slavernes øer (1970), (Les Bateaux

négriers, Actes Sud, 1996).

Heyerdahl, Thor : Kon-Tiki ekspedisjonen, 1948 (Le Voyage de Kon-Tiki).

Ibsen, Henrik : Gengangere, 1881 (Les Revenants).

Jacobsen, Roy : Seierherrene, 1991.

Nansen, Fridtjof : Paa ski over Grønland, 1890 (En ski à travers le Groenland, Hoebeke, 1996), Fram over Polhavet, 1897 (Vers le pôle, Hoebeke, 1996).

Tournier, Michel : Vendredi ou les limbes du Pacifique, 1967.

\section{RÉSUMÉS}

Dans la littérature norvégienne comme ailleurs, le motif du voyage peut servir de grille de lecture à de nombreux types d'ouvrages, notamment comme une représentation du trajet personnel parcouru par le personnage principal et comme une métaphore de la vie elle-même. Les récits de voyages héroïques qui décrivent les expéditions lancées par les Norvégiens dans des régions de conditions extrêmes, vers les pôles ou sur les mers, en particulier les récits de Fridtjof Nansen et de Thor Heyerdahl, ont en leur temps contribué à la construction nationale et - à un degré non négligeable - à l'image que les Norvégiens se font d'eux-mêmes. Le motif du voyage est encore présent dans la littérature écrite après 1970, mais quelques œuvres centrales en font un usage cette fois moins héroïque, moins optimiste.

Edvard Hoem : Kjorleikens ferjereiser (Les ferries de l'amour). Les ferries côtiers qui relient les éléments d'un littoral déchiqueté, celui de la façade atlantique de la Norvège, servent à illustrer comment les conditions matérielles déterminent autant la vie pratique des gens que leur vie sentimentale. Mette Newth : Bortførelsen (Les Voleurs d'hommes). La vie libre et communautaire des Groenlandais s'oppose à la société sinistre et inhumaine du Bergen du XVII ${ }^{\mathrm{e}}$ siècle, mais le récit fait clairement référence à la rencontre actuelle des cultures mondiales.

Erlend Loe: L. Le récit est un pastiche de récits d'expéditions héroïques, mais les " héros » ne savent dans ce cas ni ce qu'ils cherchent ni pourquoi ils le font. Le voyage sert de toile de fond à une étude ironique d'une génération de jeunes gens qui espèrent que l'avenir leur expliquera un jour qui ils étaient. Dans ces œuvres, le voyage n'est donc plus ce qu'il était, mais peut toujours servir de grille de lecture à la quête identitaire des personnages principaux.

Auch in der norwegischen Literatur kann das Reisemotiv als Leseschema für zahlreiche verschiedene Arten von literarischen Werken herangezogen werden, als Darstellung eines von der Hauptfigur zurückgelegten persönlichen Weges oder aber als Metapher für das Leben selbst. Die Erzählungen heroischer Reisen, in denen von Norwegern unternommene Expeditionen in Regionen mit extremen klimatischen Bedingungen beschrieben werden, zu den Polen oder über 
die Meere - besonders die Erzählungen Fridtjof Nansens und Thor Heyerdahls - haben zu ihrer Zeit zur nationalen Identitätsfindung beigetragen und zu einem nicht unbeträchtlichen Teil zu dem Bild, das sich die Norweger von sich selbst machen. Das Reisemotiv ist aber auch in der nach 1970 entstandenen Literatur zu finden; es wird in Enigen wesentlichen Werken verwendet, jedoch weniger heroisch und weniger optimistisch. Edvard Hoem: Kjorleikens ferjereiser (Fährfarten der Liebe). Die Küstenfähren, die die zerklüfteten Gebiete der norwegischen Atlantikküste verbinden, sind Mittel zur Darstellung, wie die materiellen Bedingungen sowohl das praktische Leben der Menschen als auch ihr Gefühlsleben bestimmen. Mette Newth: Bortførelsen (Menschenraub). Das freie und gemeinschaftsorientierte Leben der Grönländer widersetzt sich der finsteren und unmenschlichen Gesellschaft im Bergen des 17. Jahrhunderts, doch spielt die Erzählung klar auf die aktuellen Begegnungen der Weltkulturen an. Erlend Loe : $L$. Die Erzählung ist die Nachahmung von Berichten heroischer Expeditionen. Die « Helden » wissen aber in diesem Fall nicht, was sie suchen und warum sie unterwegs sind. Die Reise dient als Hintergrund für eine ironische Studie über eine Generation junger Leute, die darauf hoffen, daß ihnen die Zukunft eines Tages erklären wird, wer sie sind. In diesen Werken ist die Reise also nicht mehr das, was sie war, doch sie kann immer als Leseschema für die Identitätssuche der Hauptperson dienen.

\section{AUTEUR \\ TONE GREVE GEDDE}

Université d'Oslo 